\title{
Impaired Ventilatory Responses to Hypoxia in Mice Deficient in Endothelin-Converting-Enzyme-1
}

\author{
SYLVAIN RENOLLEAU, STÉPHANE DAUGER, GUY VARDON, BÉATRICE LEVACHER, \\ MICHEL SIMONNEAU, MASASHI YANAGISAWA, CLAUDE GAULTIER, AND JORGE GALLEGO \\ Laboratoire de Neurologie et Physiologie du Développement and Service de Physiologie, Hôpital Robert \\ Debré, Paris, France [S.R., S.D., B.L., M.S., C.G., J.G.]; Unité de Recherches sur les Adaptations \\ Physiologiques et Comportementales, Faculté de Médecine d'Amiens, Amiens, France [G.V.]; Howard \\ Hughes Medical Institute and Department of Molecular Genetics, The University of Texas Southwestern \\ Medical Center at Dallas, Dallas, Texas 75390-9050, U.S.A. [M.Y.]
}

\begin{abstract}
Endothelin-converting-enzyme (ECE-1) catalyzes the proteolytic activation of big endothelin-1 to mature endothelin-1. Most homozygous ECE-1-/- embryos die in utero and show severe craniofacial, enteric, and cardiac malformations precluding ventilatory function assessment. In contrast, heterozygous ECE$1+/-$ embryos develop normally. Their respiratory function at birth has not been studied. Taking into account previous respiratory investigations in mice with endothelin-1 gene disruption, we hypothesized that ECE-1-deficient mice may have impaired ventilatory control. We analyzed ventilatory responses to hypercapnia $\left(8 \% \mathrm{CO}_{2}\right)$ and hypoxia $\left(10 \% \mathrm{O}_{2}\right)$ in newborn and adult mice heterozygous for ECE-1 deficiency $(\mathrm{ECE}-1+/-)$ and in their wild-type littermates $(\mathrm{ECE}-1+/+)$. Ventilation, breath duration, and tidal volume were measured using whole-body plethysmography. Ventilatory responses to hypoxia were significantly weaker in ECE-1+/- than in ECE-1+/+ newborn mice (percentage ventilation increase: $1 \pm 25 \%$ versus $33 \pm 29 \%, p=$ $0.010)$. Baseline breathing variables and ventilatory responses to
\end{abstract}

\section{ABSTRACT}

hypercapnia were normal in the ECE-1+/- newborn mice. No differences were observed between adult ECE-1+/- and ECE$1+/+$ mice. We conclude that ECE- 1 is required for normal ventilatory response to hypoxia at birth. (Pediatr Res 49: 705$712,2001)$

\section{Abbreviations}

CCHS, congenital central hypoventilation syndrome

ECE-1, endothelin-converting-enzyme-1

ET-1, endothelin-1

GDNF, glial-cell-line-derived neurotrophic factor

HSCR, Hirschsprung's disease

NTS, nucleus tractus solitarius

RET, rearranged during transfection

Ттот, breath duration

VE, ventilation

$\mathbf{V}_{\mathrm{T}}$, tidal volume
ECE-1 catalyzes the proteolytic activation of big ET-1 to mature ET-1 (1, 2). Targeted ECE-1 gene disruption, which has recently been achieved in mice, has important effects on embryonic development and autonomic functions (3). Among homozygous ECE-1-/- embryos, most die in utero (about $75 \%$ in a C57BL/6-129SvEv hybrid background) and all exhibit craniofacial, enteric, and cardiac malformations (3) that preclude valid ventilatory function assessment. In contrast, heterozygous ECE-1+/- newborns are apparently healthy and develop normally. In a recent study, the differences in respiratory function between adult heterozygous ECE-1+/- and wild-type ECE-1+/+ adult mice were found to be not signif-

Received July 12, 2000; accepted December 20, 2000.

Correspondence: Dr. Jorge Gallego, Laboratoire de Neurologie et Physiologie du Développement, INSERM E9935, Hôpital Robert Debré, 48, boulevard Sérurier, 75019 Paris, France; e-mail: gallego@idf.inserm.fr

Supported by Fondation Pour La Recherche Médicale (grant awarded to S.R.) and by Université Paris VII (Legs Poix). icant (4). Newborn mice were not studied. In the present study, we tested the ventilatory responses to chemical stimuli in heterozygous ECE-1+/- newborn mice, and we reexamined the normality of adult heterozygous ECE-1+/- mice in a larger sample of mice than previously done (4).

Our working hypothesis was based on previous data showing that ET-1 is required for normal ventilatory control. First, in anesthetized rats, topical applications of ET-1 in the chemosensitive area of the ventral medulla oblongata and other areas involved in respiratory control cause changes in phrenic nerve activity (2). Specifically, applications of ET-1 in regions extending between the caudal end of the trapezoid body and the rootlets of the XIIth nerve cause transient increases followed by sustained decrease in phrenic burst rate and amplitude, whereas applications near the rootlets decrease amplitude and increase burst rate. Application of ET-1 in the NTS causes a sustained increase in phrenic nerve activity (2). Second, unanesthetized mutant mice deficient in ET-1 have impaired 
ventilatory responses to chemical stimuli. The homozygous tracheotomized newborns show practically no responses to hypercapnia and hypoxia (they die within 15-30 min if they are not tracheotomized), and the heterozygous adult mice show significantly weaker responses to chemical stimuli than their wild-type littermates (5).

On the basis of these results, we hypothesized that impaired ET-1 production in heterozygous ECE- $1+/-$ mice would translate into impaired ventilatory control. We tested this hypothesis by analyzing ventilatory responses to hypoxia and hypercapnia in ECE-1-deficient mutant mice heterozygous for the ECE-1 gene (ECE-1+/-) and in their wild-type ECE-1+/+ littermates (3).

\section{METHODS}

\section{Knockout Mice}

The colony of ECE-1-deficient mice was generated from founders provided by Dr. Yanagisawa (3). These mice were maintained as heterozygous on a C57BL/6-129SvEv hybrid background from heterozygous breed pairs (3). The mice were housed at $24^{\circ} \mathrm{C}$, with a normal 12 -h light-dark cycle, and were given food and water ad libitum. Newborn mice were obtained by mating heterozygous females with wild-type males (or the reverse) from the same C57BL/6-129SvEv hybrid background. Vaginal plugs were examined on the next morning, which was counted as embryonic d 0 (E0). Pregnant mice were killed by cervical dislocation on E18.5 post coitum (delivery normally occurs on E19). Embryos were delivered by cesarean section to control for gestational age. Each newborn was stimulated by compressing the thorax and pinching the tail. All animals of each litter were placed in a small open box with sawdust from their mother's box, at a constant ambient temperature of $32^{\circ} \mathrm{C}$ (which we found to be the mean temperature in several litters of 1-h-old newborn mice with their mother). The face of each newborn mouse was gently stimulated using a brush to mimic maternal care (6). The mean time from delivery to ventilatory testing was $59 \pm 31 \mathrm{~min}$. All the newborns survived after cesarean section until they were killed after the ventilatory tests.

We studied 56 newborn and 66 adult mice. Each animal was tested for its ventilatory response to either hypercapnia or hypoxia. In neither the newborn nor the adult populations were any significant differences in body weight or age found between the heterozygous ECE-1+/- mice and their wild-type ECE-1+/+ littermates (Table 1). The adult mice were 7-9 wk old at the time of the study. ECE- $1+/+$ and ECE- $1+/-$ adult mice had similar weights $(22.7 \pm 3.2 \mathrm{~g}$ versus $22.5 \pm 2.8 \mathrm{~g})$. Experimental protocols were approved by our institutional review board and met the animal research guidelines established by Institut National de la Santé et de la Recherche Médicale (The National Institute for Health and Medical Research).

\section{Genotyping}

Genotype was determined by PCR using DNA extracted from tail tissue (7). Primers used for the detection of PGKNeo
Table 1. Weight, age, and score in ECE-1 +/+ and ECE-1 +/newborn mice

\begin{tabular}{lcc}
\hline & ECE- $1+/+$ & ECE- $1+/-$ \\
\hline Hypoxic tests & 19 & 9 \\
Group size & $248 \pm 48$ & $248 \pm 18$ \\
Cesarean duration, s & $1.2 \pm 0.2$ & $1.2 \pm 0.1$ \\
Weight, g & $56 \pm 31$ & $62 \pm 24$ \\
Age, min & $1.7 \pm 0.5$ & $1.7 \pm 0.7$ \\
Score & 15 & \\
Hypercapnic tests & $253 \pm 32$ & 13 \\
Group size & $1.2 \pm 0.2$ & $230 \pm 55$ \\
Cesarean duration, s & $66 \pm 37$ & $54 \pm 29$ \\
Weight, g & $1.6 \pm 1.0$ & $1.3 \pm 1.0$ \\
Age, min & & \pm 0.1 \\
Score & & \\
\hline
\end{tabular}

Values are means \pm SD. Differences between ECE-1 +/+ and ECE-1 +/groups were not significant. Gender has no significant effect on weight or score.

were downstream, 5'-TATTCGGCTATGACTGGGCACAACAG-3' and upstream 5'-TTCCACCATGAT ATTCGGCA AGCAGG-3'. The amplification program comprised 35 cycles, each consisting of $3 \mathrm{~min}$ at $94^{\circ} \mathrm{C}, 1 \mathrm{~min}$ at $94^{\circ} \mathrm{C}, 1 \mathrm{~min}$ at $55^{\circ} \mathrm{C}$, and 2 min at $72^{\circ} \mathrm{C}$. PCR products were analyzed in $1 \%$ agarose gel.

\section{Determination of Newborn Gender}

Newborn gender was determined by PCR detection of the chromosome Y sry gene (7). Primer sequences were 5'GAGAGCATGGAGGGCCAT-3' and 5'-CCACTCCTCTGTG TACACT-3'. The amplification program comprised 30 cycles of $30 \mathrm{~s}$ at $96^{\circ} \mathrm{C}, 30 \mathrm{~s}$ at $57^{\circ} \mathrm{C}$, and $30 \mathrm{~s}$ at $72^{\circ} \mathrm{C}$. PCR products were analyzed in $1 \%$ agarose gel.

\section{Ventilatory Measurements}

Ventilatory parameters in newborn and adult mice were obtained noninvasively using whole-body, barometric plethysmography, based on Drorbaugh and Fenn's principle (8-12). According to this principle, when an animal breathes in a nondistensible chamber, the pressure in the chamber increases during inspiration owing to addition of water vapor to the inspired gas and to warming of the inspired gas from the temperature in the chamber to that in alveoli; conversely, pressure decreases during expiration owing to condensation of water vapor and cooling of expired gas. Measurement of these pressure variations can be used to calculate Ттот, $\mathrm{V}_{\mathrm{T}}$, and $\mathrm{V}_{\mathrm{E}}$ (calculated as $\mathrm{V}_{\mathrm{T}} / \mathrm{T}$ тот).

Newborn mice. The plethysmograph used for newborn mice has been described previously (13). Briefly, the system was composed of two $30-\mathrm{mL}$ syringes serving as measurement and reference chambers, respectively. The syringes were immersed in a thermoregulated water bath that maintained the temperature inside the syringes between 28 and $30^{\circ} \mathrm{C}$. Leakage through a catheter (time constant, 2 s) avoided slow pressure drifts between the two syringes. The pressure difference between the measurement and the reference chambers (EFFA pressure transducer, Asnières, France; range $\pm 0.1 \mathrm{mb}$ ) was filtered 
(bandwidth, $0.05-15 \mathrm{~Hz}$ at $-3 \mathrm{~dB}$ ), converted to a digital signal (MacAdios A/D 12-bits converter, GW-Instruments, Somerville, MA, U.S.A.) at a sample rate of $100 \mathrm{~Hz}(14-18)$, and used by a computer (PowerWave 604/150) to calculate Ттот (ms), Vт (divided by body weight, $\mu \mathrm{L} / \mathrm{g})$, and $\mathrm{V}_{\mathrm{E}}(\mu \mathrm{L} / \mathrm{s} / \mathrm{g})$ (Software Superscope II, GW-Instruments, Somerville, MA, U.S.A.).

Calibration was done before each test by injecting $5 \mu \mathrm{L}$ of air into the measurement chamber and introducing the corresponding pressure into Drorbaugh and Fenn's equation. The pressure rise in the measurement chamber induced by this injection was of similar magnitude to that induced by the $V_{\text {т }}$ of a newborn mouse. To avoid restraining the animals, body temperature was not recorded and was assumed to be stable at $33^{\circ} \mathrm{C}$ in the calculation of Vт from Drorbaugh and Fenn's equation. Only dry gases were introduced into the measurement chamber to minimize errors in $\mathrm{V}_{\text {т }}$ calculations due to inaccurate estimation of vapor pressure, and therefore we assumed in the $\mathrm{V}_{\text {т }}$ calculation from the Drorbaugh and Fenn equation that relative humidity was near zero. Mouth temperatures in the newborn mice are not reported because we detected a failure in the calibration of the thermistor probe after we completed the study (however, mouth temperature was roughly estimated at $\left.33^{\circ} \mathrm{C}\right)$. Hypercapnic $\left(8 \% \mathrm{CO}_{2}, 21 \% \mathrm{O}_{2}\right.$, and $\left.71 \% \mathrm{~N}_{2}\right)$ and hypoxic $\left(10 \% \mathrm{O}_{2}, 3 \% \mathrm{CO}_{2}\right.$, and $\left.87 \% \mathrm{~N}_{2}\right)$ mixtures were introduced into the measurement chamber through a catheter (5-mL dead space). The hypoxic mixture contained 3\% $\mathrm{CO}_{2}$ to approach isocapnia during hypoxic stimulus (19).

Adult mice. The plethysmograph for adult mice has been described in detail elsewhere $(13,14)$. It was composed of three superimposed, cylindrical Plexiglas chambers with a capacity of $0.6 \mathrm{~L}, 3.6 \mathrm{~L}$, and $3.6 \mathrm{~L}$, respectively. The upper chamber was for gas admission and mixing, the middle chamber served as a reference for pressure measurements, and the lower chamber contained the animal. A $1500 \mathrm{~mL} / \mathrm{min}$ flow of dry air (Bronkhorst Hi-Tec airflow stabilizer, Uurlo, Holland) was delivered through the admission chamber and subsequently divided into two $750 \mathrm{~mL} / \mathrm{min}$ flows through the reference and measurement chambers, respectively. The pressure difference between the reference and measurement chambers was determined (EFFA pressure transducer, range $\pm 0.1 \mathrm{mb}$ ), filtered (bandwidth, $0.4-15 \mathrm{~Hz}$ at $-3 \mathrm{~dB}$ ), and converted into a digital signal. The plethysmograph was calibrated before each experiment by injecting $100 \mu \mathrm{L}$ of air into the measurement chamber. Body temperature was not recorded and was assumed to be stable at $37^{\circ} \mathrm{C}(15-18)$. Fractional $\mathrm{CO}_{2}$ and $\mathrm{O}_{2}$ concentrations in the measurement chamber were determined continuously (Arelco $\mathrm{CO}_{2} / \mathrm{O}_{2}$ analyzer, Fontenay-sous-Bois, France) by sampling $100 \mathrm{~mL} / \mathrm{min}$ from the plethysmograph outflow. The ambient temperature inside the measurement chamber during ventilatory measurements was $25.0 \pm 2.3^{\circ} \mathrm{C}$. The mean temperature increase inside the measurement chamber was $<0.6^{\circ} \mathrm{C}$, a value that has been shown to cause a $<1 \%$ error in $\mathrm{V}_{\text {т }}$ measurements (9). The water partial pressure in the barometric chamber (which entered into Drorbaugh and Fenn's equation for $\mathrm{V}_{\mathrm{T}}$ calculation) was assumed to be kept at zero by the dry gas flushing the plethysmograph. However, direct measurement of relative humidity in the chamber caused by the water content of expired gas and urine indicated that this variable may increase up to $15 \%$, thus leading to a $\mathrm{V}_{\text {т }}$ measurement error of $<4 \%$ (9). In a previous study in Swiss adult mice (one restrained mouse and one unrestrained mouse), telemetric temperature recordings showed that the magnitude of temperature changes during the air, hypercapnic, and hypoxic tests was $<0.7^{\circ} \mathrm{C}$. These changes had a negligible effect on $\mathrm{V}_{\mathrm{T}}$ calculations (9).

\section{Procedure}

Newborn mice. Adaptation to extrauterine life was assessed by scoring the following criteria on a three-point scale (0 to 2): color (marked cyanosis, mild cyanosis, or pink, respectively), breathing (apnea, irregular breathing, regular breathing), spontaneous motor activity (no movement, weak movements, or vigorous movements), and reactivity to tail pinching (no response, movement, or movement and vocalization). These four scores were summed (Table 1). The ventilatory tests were started after $1 \mathrm{~min}$ of familiarization inside the measurement chamber. Each test consisted of five successive steps: 1) syringe flushing with $60 \mathrm{~mL}$ of air; 2) breathing variable recording for $90 \mathrm{~s} ; 3$ ) syringe flushing with $60 \mathrm{~mL}$ of air; 4) injection of the hypercapnic or hypoxic mixture; and 5) breathing variable recording for $90 \mathrm{~s}$. Each gas injection took about $45 \mathrm{~s}$. Pressure signals became available about $15 \mathrm{~s}$ after the end of each gas injection because of the transient pressure signal disturbance caused by the injection. Each animal underwent two identical hypoxic or hypercapnic tests. After the last recording, the newborn was removed from the syringe and weighed. It was then killed by neck section, and a piece of its tail was collected for genotyping and gender determination.

Adult mice. The heterozygous and wild-type groups were matched on gender, time of the experiment (morning, midday, or evening), and order of stimuli (hypercapnia first or hypoxia first). The mice were tested under loose restraint according to a previously described protocol $(13,14)$. Each mouse was weighed and placed in a cylindrical, wire-mesh cage $(9 \mathrm{~cm}$ long by $3.5 \mathrm{~cm}$ in diameter) to limit movement artifacts. After a 30-min familiarization period, the airflow through the plethysmograph was replaced by a hypercapnic or hypoxic flow (with the same compositions as for the newborns: $8 \% \mathrm{CO}_{2}$, $21 \% \mathrm{O}_{2}$, and $71 \% \mathrm{~N}_{2}$ or $10 \% \mathrm{O}_{2}, 3 \% \mathrm{CO}_{2}$, and $87 \% \mathrm{~N}_{2}$ ). The switch from air to the hypoxic or hypercapnic mixture was controlled by the computer via electrovalves. The gas mixtures were flushed into the plethysmograph for 15 min. Steady gas concentrations were obtained $10 \mathrm{~min}$ after electrovalve opening.

Each mouse received two consecutive hypoxic tests and two consecutive hypercapnic tests. In each group (heterozygous or wild-type), half of the mice had the two hypoxic tests first, and then the two hypercapnic tests, and the other half received the test in the reversed order. Each test consisted of a 15-min air period and a 15-min stimulus period (the time lapse between the two exposures to hypoxia or hypercapnia was therefore 15 min). Because of the residual $\mathrm{CO}_{2}$ level inside the plethysmograph $(0.5 \%)$, the fractional $\mathrm{CO}_{2}$ concentration was $8.5 \%$ 
during hypercapnic stimulation and 3.5\% during hypoxic stimulation.

\section{Data Analysis}

Newborn mice. Ventilatory data free from movement artifacts were selected visually by discarding tracing segments without individualized breaths or with drifts larger than twice the mean amplitude of the volume signal. After averaging Ттот, Vт, and $\mathrm{V}_{\text {E }}$ over each valid segment, we calculated the overall mean of these average values weighted for the number of breaths in each segment. Although data sample size was sufficient for a valid estimation of breathing variables, the occurrence of movement artifacts and the brevity of gas exposure (90 s) precluded a fine-tuned analysis of the time course of the hypoxic and hypercapnic responses.

To control for possible carry-over effects of the first stimulus on the second air period $(20,21)$, we used the first air period as the baseline. Data from the two stimulation periods were averaged. Apneas were defined as ventilatory pauses longer than twice the mean duration of the immediately preceding valid breath. Apnea number and mean duration were determined individually for each air and each stimulus period.

Adult mice. Ттот, $\mathrm{V}$, and $\mathrm{V}_{\mathrm{E}}$ were averaged over successive 15 -s periods during the last 3 min of each phase. Ventilation consistently increased as a function of time (no ventilatory decline was observed during the hypoxic stimulus). The mean values during the last $3 \mathrm{~min}$ of stimulus represented the peak value of the response. All the values of 3-min period were entered into the analysis.

\section{Statistics}

We performed separate ANOVA for newborns and adults and for each stimulus (hypoxia and hypercapnia) and variable (Ттот, Vт, and $\mathrm{V}_{\mathrm{E}}$ ) using a statistical software (Superanova, Abacus Concepts, Berkeley, CA, U.S.A.). In all analyses, the two between-subject factors were genotype (two levels: heterozygous ECE-1+/-versus wild-type ECE-1+/+) and gender. Gas (two levels: air versus hypercapnia or air versus hypoxia) was the within-subject factor. In addition, age (in minutes) was a cofactor in the newborns, and testing time (three levels: morning, midday, and afternoon) and stimulus order (two levels: hypercapnia followed by hypoxia or the reverse) were between-subject factors in the adults.

The main effect for gas reflected the ventilatory response to a given stimulus. Consequently, the differences between heterozygous ECE-1+/- and wild-type ECE- $1+/+$ mice with respect to ventilatory response were evaluated based on the group-by-gas interaction. We also used percentage changes from normoxic values as indices of ventilatory responses to hypoxia and hypercapnia. Data are summarized as the group means $\pm \mathrm{SD}$ in the text and as the means \pm SEM in the figures. $F$ ratios are indicated along with the $d f$ of the numerator and the denominator. Differences were considered significant when $p$ $<0.05$.

\section{RESULTS}

\section{Newborn Mice}

There were no significant differences between ECE-1+/+ and ECE-1+/- for the following variables: scores (Table 1); baseline values of $\mathrm{VE}_{\mathrm{E}}, \mathrm{V}_{\mathrm{T}}$, and Tтот, the number of breaths used for the calculation of averaged breathing variables (Table 2 ); and number, mean duration, and cumulated time of apneas $(1 \pm 1$ versus $2 \pm 2 ; 1.8 \pm 2.0 \mathrm{~s}$ versus $2.4 \pm 1.7 \mathrm{~s}$, and 3.9 $\pm 5.8 \mathrm{~s}$ versus $5.2 \pm 5.2 \mathrm{~s}$, respectively).

Ventilatory responses to hypoxia and hypercapnia in newborn mice are shown in Figure $1, A$ and $B$. ECE- $1+/+$ newborn mice increased $V_{E}$ in response to hypoxia $[33 \pm 29 \%$, $F(1,18)=15.18, p<0.001]$ by increasing $\mathrm{V}_{\mathrm{T}}[25 \pm 34 \%, F$ $(1,18)=4.63, p<0.05]$ and decreasing Ттот $[-9 \pm 19 \%, F$ $(1,18)=5.77, p<0.03$, Fig. $1 A]$. In contrast, ECE- $1+/-$ newborn mice showed virtually no change in $V_{E}$ in response to hypoxia $(1 \pm 24 \%)$; neither the change in $\mathrm{V}_{\mathrm{T}}(11 \pm 22 \%)$ nor the change in Ттот $(12 \pm 19 \%)$ was significant, suggesting that both components of the response to hypoxia were impaired. Illustrative examples of plethysmographic recordings in ECE-1 $+/+$ and ECE-1 $+/-$ newborns are shown in Figure 2. The differences between ECE-1+/+ and ECE-1+/- were confirmed by the demonstration of significant group-by-gas interactions for $\mathrm{VE}[F(1,26)=6.60 ; p=0.016]$ and Tтот $[F(1$, $26)=5.86 ; p=0.023]$ but not VT. Analysis of the percentage of variation from air to hypoxia and the above comparisons between air and hypoxia yielded the same statistical results. Finally, there were no significant differences between ECE-1+/+ and ECE-1+/- newborns in apnea number or in apnea duration during hypoxia.

We found no significant differences in VE response to hypercapnia or in apnea number or duration during hypercapnia between the ECE-1+/+ and ECE-1+/- newborns (VE percentage increase, $29 \pm 42 \%$ versus $25 \pm 39 \%$, respectively; Fig. $1 B)$.

Gender or age had no significant effect on breathing variables, either as a main factor or as a source of interaction with other factors.

\section{Adult Mice}

No significant differences in $\mathrm{VE}_{\mathrm{E}}, \mathrm{V}$, or Tтот were found between ECE-1+/+ and ECE-1+/- adult mice during base-

Table 2. Baseline ventilatory variables in newborn and adult mice

\begin{tabular}{ccc}
\hline & ECE- $1+/+$ & ECE- $1+/-$ \\
\hline Newborn mice & & \\
TToт, ms & $630 \pm 281$ & $671 \pm 199$ \\
VT, $\mu \mathrm{L} / \mathrm{g}$ & $3.1 \pm 2.0$ & $3.9 \pm 2.4$ \\
VE $\mu \mathrm{L} / \mathrm{g} / \mathrm{s}$ & $5.3 \pm 2.2$ & $6.0 \pm 2.9$ \\
Adult mice & & \\
TTот, ms & $390 \pm 49$ & $382 \pm 59$ \\
VT, $\mu \mathrm{L} / \mathrm{g}$ & $7.1 \pm 1.0$ & $7.1 \pm 1.0$ \\
VE, $\mu \mathrm{L} / \mathrm{g} / \mathrm{s}$ & $19.0 \pm 3.1$ & $19.4 \pm 3.8$ \\
\hline
\end{tabular}

Baseline data were averaged over the air periods preceding the hypercapnic or the hypoxic tests. Values are means \pm SD. The number of breaths used for the calculation of averaged breathing variables in newborn ECE- $1+/+$ and ECE-1+/- were $69 \pm 47$ and $50 \pm 10$, respectively. Differences of baseline data between ECE-1 +/+ and ECE-1 +/- groups were not significant. 

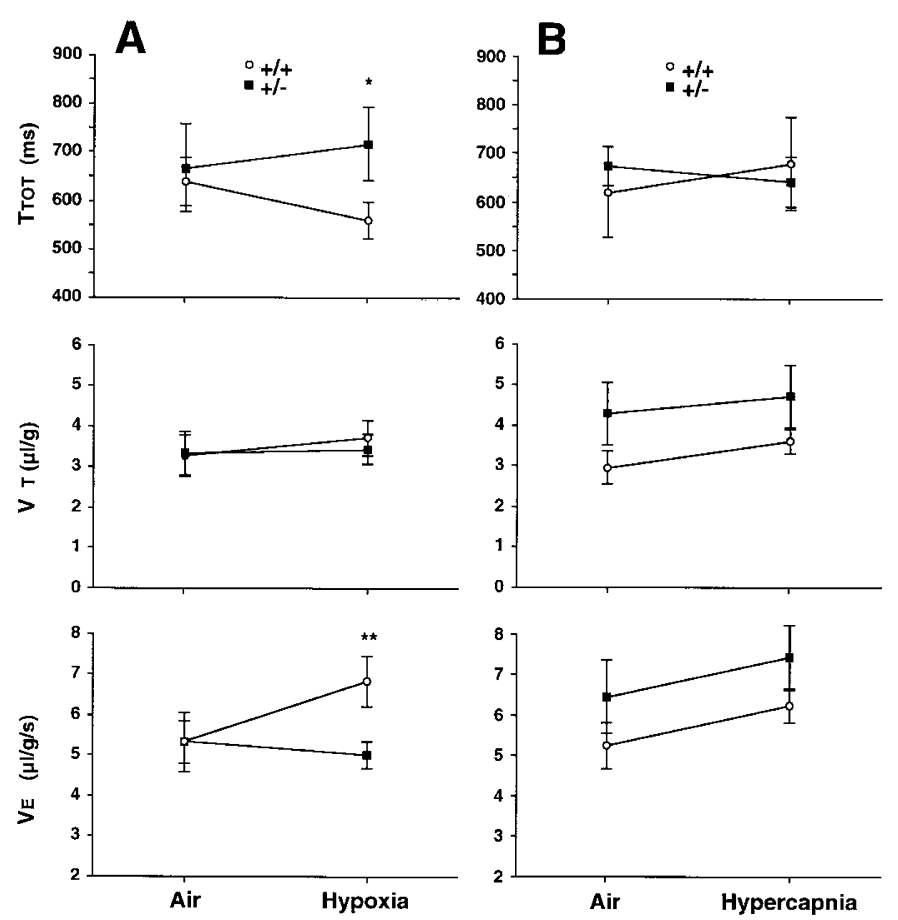

Figure 1. Ventilatory responses to hypoxia (panel $A$ ) and hypercapnia (panel $B)$ in newborn mice. Heterozygous ECE- $1+/-$ mice $(n=9)$ had significantly weaker responses to hypoxia than wild-type ECE- $1+/+$ mice $\left(n=19 ;{ }^{* *} p=\right.$ $0.016 ; * p=0.023)$. Ventilatory responses to hypercapnia were not significantly different between heterozygous ECE-1+/- newborn $(n=13)$ and wild-type ECE- $1+/+$ newborn $(n=15)$ mice. Values are means \pm SEM.

line ventilation (Table 2). Baseline $\mathrm{VE}_{\mathrm{E}}, \mathrm{V}_{\mathrm{t}}$, and Tтот values were higher in females than in males, but these differences were significant for $\mathrm{V}_{\text {т }}$ only [main effect for gender, $F(1,64)$ $=28.57 ; p<0.0001]$. The gender-by-genotype interaction was not significant.

No significant differences were demonstrated in the ventilatory responses to hypoxia or hypercapnia between the ECE- $1+/+$ and ECE- $1+/-$ adult mice (hypoxic VE response, $81 \pm 41 \%$ versus $75 \pm 32 \%$; hypercapnic VE response, $127 \pm 61 \%$ versus $130 \pm$

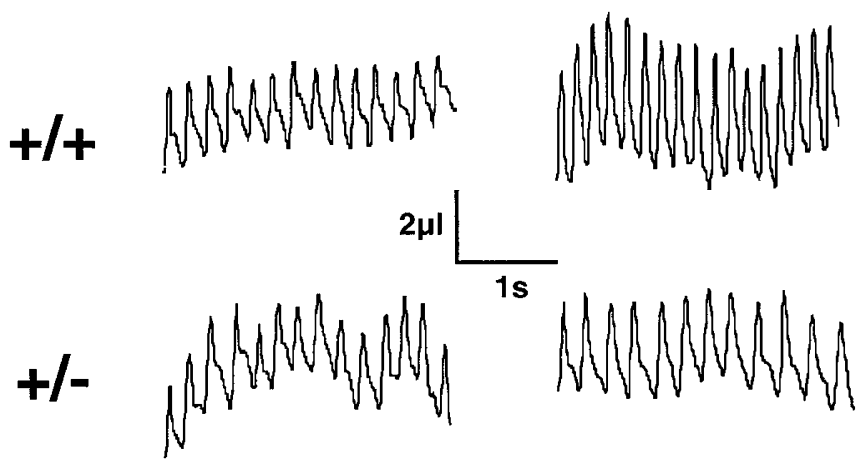

AIR

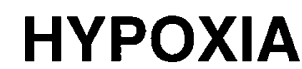

Figure 2. Individual ventilatory responses to hypoxia in two representative newborn mice, one ECE-1+/+ and the other ECE-1+/-. Portions of the volume signal obtained by whole body plethysmography. Baseline ventilation was similar in the two mice. The wild-type ECE-1+/+ newborn responded to hypoxia by increasing $\mathrm{V}_{\mathrm{T}}$ and decreasing Ттот, whereas these variables showed minimal changes in the heterozygous ECE-1+/- newborn.
$74 \%$, respectively, Fig. 3, $A$ and $B$ ). Gender had no significant effects on these responses, either as a main effect or as a source of interaction with other factors.

\section{DISCUSSION}

The results of this study show that heterozygous ECE-1+/newborn mice had a significantly weaker ventilatory response to hypoxia than their wild-type ECE- $1+/+$ littermates. The ventilatory response to hypercapnia was normal in heterozygous ECE-1+/- newborns, demonstrating that their impaired response to hypoxia was not caused by a limitation in their ability to increase ventilation. These results support the hypothesis that the ECE-1 gene may be required for normal ventilatory response to hypoxia at birth. The ventilatory responses to hypoxia and hypercapnia were not significantly different between the heterozygous and the wild-type adult mice investigated while loosely restrained.

\section{Animals}

We found no significant differences between the heterozygous ECE-1+/- newborn mice and their wild-type ECE-1+/+ littermates in terms of extrauterine life adaptation scores, weight, or baseline breathing pattern. These data are in line with previous reports that heterozygous ECE- $1+/-$ newborns developed normally, in striking contrast to homozygous ECE-1-/- mice (3). These previous studies showed that about $75 \%$ of ECE-1-/- embryos died in utero in a C57BL/6-129SvEv hybrid background (3). The comparison of the observed number of ECE-1-/- newborns and the number expected based on the Mendelian distribution suggested that the in utero death rate was about $75 \%$ in the present study also.
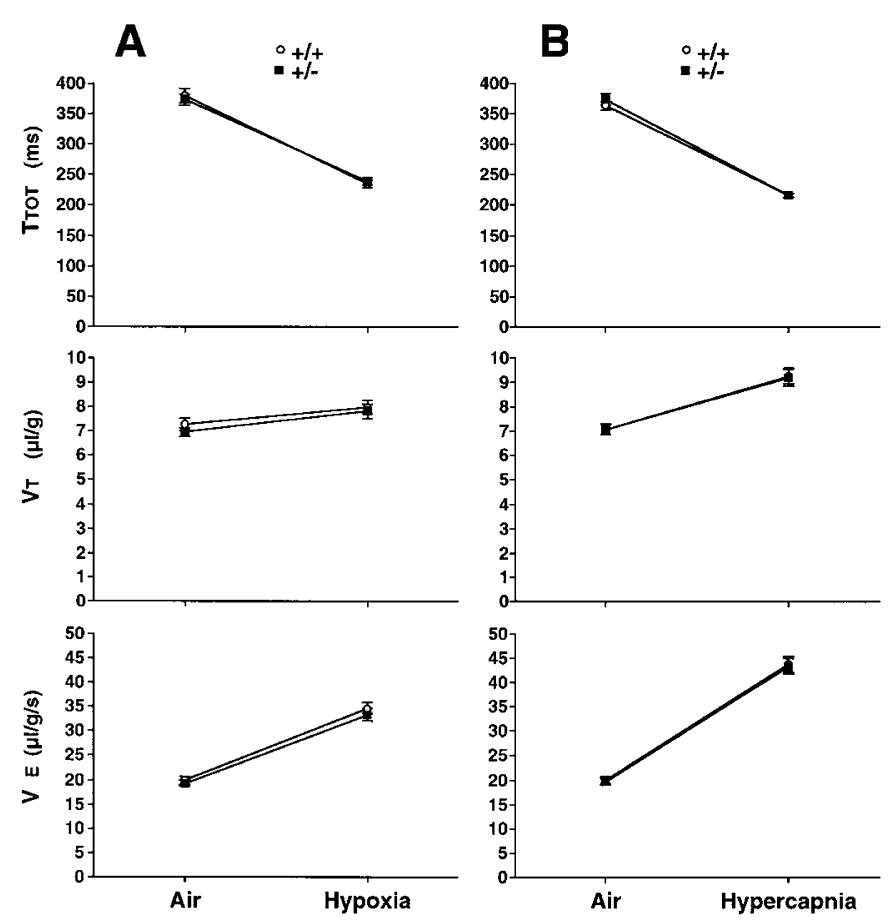

Figure 3. Ventilatory responses to hypoxia (panel $A$ ) and hypercapnia (panel $B)$ in adult mice. Ventilatory responses to hypoxia and hypercapnia were not significantly different between heterozygous ECE-1+/- $(n=37)$ and wildtype ECE- $1+/+(n=29)$ adult mice. Values are means \pm SEM. 
Previous studies also showed that live-born homozygous ECE-1-/mice were cyanotic and exhibited major craniofacial and cardiovascular malformations, aganglionic megacolon, and pigment disorders; they died within $30 \mathrm{~min}$ after birth (3). Their birth defects reflected disruption of distinct sets of neural crest derivatives by the ECE-1 mutation. The craniofacial malformations preclude ventilatory testing. Homozygous ET-1-/- newborn mice with similar craniofacial malformations have been tested using tracheostomy (5), but not all these animals exhibit the cardiac malformations and circulatory failure that are consistently present in homozygous ECE-1-/- newborn mice (3) and preclude a valid assessment of ventilatory function.

\section{Plethysmographic Measurement Validity}

Our data were generated by a comparison of breathing variables (Ттот, $\mathrm{V}_{\mathrm{T}}$, and $\mathrm{VE}_{\mathrm{E}}$ ) between air and hypoxia or hypercapnia. ANOVA was used to test for differences between these two conditions, and these differences were compared between heterozygous and wild-type mice. We also calculated the percentage change (i.e. the ratio) from air to gas stimulus and compared this ratio between groups. In the present study, these two approaches produced identical statistical results: the ventilatory response to hypoxia was weaker in the heterozygous ECE-1+/- newborns.

Our finding that the ECE-1+/- newborns had weaker ventilatory responses to hypoxia than their ECE-1+/+ littermates could be ascribed to the fact that neither the change in $\mathrm{V}_{\mathrm{T}}$ nor the change in Ттот was significant. This fact deserves attention because the accuracy of $V_{T}$ calculations based on whole-body plethysmography data has been questioned $(11,12)$. In adult mice, the comparison of $V_{T}$ calculations from whole-body plethysmography data and pneumotachography data has shown a $<7 \%$ difference (12), suggesting that whole-body plethysmography may provide accurate $\mathrm{V}_{\mathrm{T}}$ values. In newborn mice, reference $V_{T}$ values are difficult to obtain, for technical reasons. Several sources of error, such as body temperature and chamber temperature and humidity are difficult to control in small devices. These difficulties undoubtedly explain the large variability of data obtained in previous studies, as discussed previously (13). Therefore, the absolute $\mathrm{V}_{\mathrm{T}}$ and $\mathrm{VE}_{\mathrm{E}}$ values obtained in our newborns should be viewed with caution. However, they are similar to those previously reported in newborn mice with the same genetic background (13).

\section{The Impaired Response to Hypoxia in ECE-1+/- Newborns}

Heterozygous ECE-1+/- newborns did not differ from wild-type newborn mice during room air breathing. Their ventilatory response to hypercapnia was in the range of wildtype values, suggesting that their respiratory mechanics were not impaired. The correction achieved by adding $3 \% \mathrm{CO}_{2}$ to the hypoxic mixture as performed in this study and in previous studies by other authors in adult mice $($ e.g. 17, 18) has not been validated in newborn mice based on blood gas analysis. We cannot exclude that the $3 \% \mathrm{CO}_{2}$ concentration overcompensated the hypoxia-induced hypocapnia, and therefore that the hypoxia-induced change in $\mathrm{VE}_{\mathrm{E}}$ observed in the newborn mice was in fact a response to hypercapnic hypoxia. However, this is unlikely inasmuch as the $\mathrm{VE}$ response to $10 \% \mathrm{O}_{2}+3 \% \mathrm{CO}_{2}$ was barely detectable and the response to hypercapnia was unimpaired, as shown by the hypercapnic test itself. Therefore, the uncertainty about the extent to which normocapnia was maintained during hypoxia does not detract from our finding that the response to hypoxia was impaired in the ECE-1+/newborns.

The typical time course of the response to hypoxia, which generally shows a peak-value followed by a decline in newborn mammals (22), could not be examined in the present study for the following three reasons: 1) the initial transient instability of the pressure signal caused by the injection of the hypoxic mixtures into the measurement chamber, 2) the occurrence of movement artifacts, and 3) the relatively short duration of the exposure to hypoxia $(90 \mathrm{~s})$. The data free from measurement and movement artifacts, which were visually selected, did not necessarily correspond to the peak value of ventilation, thus leading to a possible underestimation of the response to hypoxia. This methodological limitation did not invalidate our claim that this response was impaired in ECE-1+/- newborn. However, our method did not allow determination of whether this result was due to a smaller peak response to hypoxia or a stronger decline of this response. Furthermore, during early development, there is a shift from dominance of the central inhibitory component of the response to hypoxia, to dominance of the peripheral excitatory component of this response (23). The time course of this process has not been examined in mice, but recent data from our laboratory indicate that chemoreceptor resetting in mice occurs around $12 \mathrm{~h}$ after birth, and that the response to hypoxia is small before this age. Therefore, the newborn animals in the present study were tested at an age (on average $1 \mathrm{~h}$ after birth) characterized by immaturity of the response to hypoxia. This explains the relatively small $\mathrm{VE}_{\mathrm{E}}$ increase in all the animals, including the wild-type ECE-1+/+ newborns. Delayed maturation of the response to hypoxia in ECE-1+/- mice could theoretically account for their impaired response to hypoxia. Further assessments of the ventilatory response to hypoxia should include an analysis of the time course of this response. In addition, the hyperoxic test, which consists of measuring the decrease in minute ventilation caused by sudden inhalation of pure oxygen (physiologic chemodenervation), may allow assessment of the respiratory drive from oxygen-sensitive receptors. Both analysis of the hypoxic decline and the hyperoxic test may shed light on the mechanisms of the abnormal response to hypoxia in ECE- $1+/-$ newborn mice.

The impaired response to hypoxia in our heterozygous ECE-1+/- newborns may be ascribable to low ET-1 levels. Heterozygous ECE-1+/- embryos have been reported to exhibit weaker ECE-1 activity and lower endothelin levels than wild-type ECE-1+/+ embryos, although the second difference was not significant in the small groups of embryos studied (3). Endothelin peptides and receptors are found at many sites within the central and peripheral nervous systems, in particular those controlling the ventilatory response to hypoxia, i.e. the oxygen-sensitive chemoreceptors in the carotid bodies and their projections to the NTS and ventrolateral medulla oblongata via the carotid sinus nerve. Accordingly, reduced conver- 
sion of big ET-1 to mature ET-1 at these sites might account for the impaired response to hypoxia seen in our ECE-1+/newborn mice. In addition, the ventilatory response to hypoxia may also be mediated by chemosensitive cells in the aortic bodies and by sensory fibers in the abdominal vagus nerve (24, 25 ). Oxygen-sensitive neurons located in the caudal hypothalamus also modulate the ventilatory response to hypoxia, even in the absence of input from the vagus nerve and carotid sinus nerve (26). This may be important because the hypothalamus and medulla oblongata are the sites of highest ECE-1 activity in the CNS (2). Therefore, reduced ECE-1 levels at these sites may also result in an impaired response to hypoxia, even when the peripheral chemoreceptors are normal. Finally, we cannot rule out the possibility that our findings were produced by an abnormality in the arousal response to hypoxia (27, not investigated in the present study), which is mediated by other important sites with ET-1 activity, such as cortical areas, subcortical areas, and the hypothalamus (2).

\section{Normal Responses to Hypoxia and to Hypercapnia in Adult Mice}

Baseline ventilatory variables and ventilatory responses to hypoxia and hypercapnia were not significantly different between adult wild-type ECE-1+/+ and adult heterozygous ECE-1+/- mice. Conceivably, impairment of ventilatory responses to hypoxia in heterozygous ECE-1+/- newborn mice may be overcome during development. This interpretation is supported by the fact that endothelin expression in the CNS increases considerably with age. For example, in rat brain, the ET-1 concentration at full term (i.e. E21) is about 2-fold that at E18.5 (28), and ET-1 concentrations in the medulla oblongata and hypothalamus increase between 1 and 8 wk of age (29). Therefore, the ontogenetic development of ET-1 expression may compensate for the impairment seen in the neonatal period. However, this interpretation is at variance with previous evidence that ET- $1+/$ - adult mice have slight but significant impairments of the responses to hypoxia and hypercapnia (5). Alternatively, the ECE-1+/- mice that survived to adulthood may have been those with the mildest ventilatory impairment. Only a longitudinal study of morbidity in heterozygous ECE-1+/- mice can clarify this issue.

Finally, the normal response to hypoxia in heterozygous ECE-1+/ - adult mice may be ascribable to our use of loose restraint to limit movement artifacts during the tests in adult but not in newborn mice. Despite its potent arousing effects, the restraint method used in the present study [similar to that used in Kuwaki et al. 's experiment (5)] has been reported to be devoid of significant effects on responses to hypoxia or hypercapnia in normal mice (14). Possibly, the impaired response to hypoxia in the ECE-1+/- newborns was ascribable to an abnormal arousal response to hypoxia. Under this hypothesis, the normal response to hypoxia in the adult ECE-1+/+ may reflect restraint-induced arousal. This hypothesis could be tested by specifically evaluating the arousal response to weaker hypoxic stimuli in sleeping, unrestrained, adult mice.

\section{Involvement of Neural Crest Derivatives in Early Impairments of Ventilatory Control}

The present data were obtained as part of a recently started research effort aimed at identifying genes involved in the development of the chemical control of breathing. This control is sensitive to mutations in genes involved in neural crest development $(4,5,13,30,31)$, genes encoding trophic factors $(32,33)$, and genes encoding enzymes involved in neurotransmitter production or breakdown $(34,35)$. Some of these mutations are associated with lethal respiratory failure in newborn null mutants. This is the case for Ret (30), Mash-1 (13), ET-1 (5), and ET-A (4). Most available data suggest that development of chemosensitivity is controlled by multiple genes and involves various, probably interdependent signaling pathways.

Our findings point toward a possible role of ECE- 1 gene in early impairments of chemosensitivity in humans, particularly the CCHS. The respiratory phenotype of CCHS is characterized by central alveolar hypoventilation during sleep, and also during wakefulness in the most severe cases. There are no ventilatory responses to hypercapnia or hypoxia in these patients, and this impairment in chemosensitivity is lifelong (36). The severely depressed response to hypoxia in the ECE-1+/newborn mice as compared with their ECE- $1+/+$ littermates is reminiscent of the impaired chemosensitivity to hypoxia in CCHS patients. However, the ventilatory response to hypercapnia - which is impaired in CCHS patients-was normal in the ECE-1+/- newborn mice. Furthermore, the normal response to hypoxia in the adult ECE-1+/- mice is an important phenotypic difference with CCHS.

A recent report of an ECE-1 mutation with loss of function in a patient with HSCR and autonomic dysfunction (37) is interesting in this respect, because $20 \%$ of reported CCHS patients also had HSCR, suggesting a common pathogenic process. Several genes involved in the development of neural crest derivatives (e.g. ECE-1) have been shown to be involved in CCHS and/or HSCR. Mutations in RET or its ligands (GDNF and neurturin) have been reported in two cases of CCHS, one with and one without $\operatorname{HSCR}(38,39)$. In addition, a mutation in endothelin-3 has been found in one patient with CCHS (40). These clinical data support the hypothesis that genes involved in the development of neural crest lines and belonging to several signaling pathways, including the endothelin pathway, may be required for normal development of ventilatory control. The involvement of ECE-1 in the development of the ventilatory response to hypoxia provides further support for this hypothesis and suggests that scanning the human ECE-1 gene (41) for mutations in patients with CCHS may shed light on the genetic causes of this disease.

\section{REFERENCES}

1. Xu D, Emoto N, Giaid A, Slaughter C, Kaw S, de Wit D, Yanagisawa M 1994 ECE-1 a membrane-bound metalloprotease that catalyzes the proteolytic activation of big endothelin-1. Cell 78:473-485

2. Kuwaki TH, Kurihara H, Cao WH, Kurihara Y, Unekawa M, Yazaki Y, Kumada M 1997 Physiological role of brain endothelin in the central autonomic control: from neuron to knockout mouse. Prog Neurobiol 51:545-579

3. Yanagisawa H, Yanagisawa M, Kapur RP, Richardson JA, Williams SC, Clouthier DE, de Wit D, Emoto N, Hammer R 1998 Dual genetic pathways of endothelinmediated intercellular signaling revealed by targeted disruption of endothelin converting enzyme-1 gene. Development 125:825-836 
4. Kuwaki L, Ling GY, Onodera M, Ishii T, Nakamura A, Ju KH, Cao WH, Kumada M, Kurihara H, Kurihara Y, Yazaki Y, Ohuchi T, Yanagisawa M, Fukuda Y 1999 Endothelin in the central control of cardiovascular and respiratory functions. Clin Exp Pharmacol Physiol 26:989-994

5. Kuwaki T, Cao WH, Kurihara Y, Kurihara H, Ling GY, Onodera M 1996 Impaired ventilatory responses to hypoxia and hypercapnia in mutant mice deficient in endothelin-1. Am J Physiol 270:R1279-R1286

6. Ronca AE, Abel RA, Alberts JR 1996 Perinatal stimulation and adaptation of the neonate. Acta Paediatr 416:8-15

7. Hogan B, Beddington R, Constantini F, Lacy E 1994 Manipulating the Mouse Embryo: Laboratory Manual. Cold Spring Harbor Laboratory Press, Plainview, NY, pp 1-497

8. Drorbaugh JE, Fenn WO 1955 A barometric method for measuring ventilation in newborn infants. Pediatrics 16:81-86

9. Epstein MAF, Epstein RA 1978 A theoretical analysis of the barometric method for measurement of tidal volume. Respir Physiol 32:105-120

10. Mortola JP, Frappell PB 1998 On the barometric method for measurements of ventilation, and its use in small animals. Can J Physiol Pharmacol 76:937-944

11. Enhorning G, van Schaik S, Lundgren C, Vargas I 1998 Whole-body plethysmography, does it measure tidal volume of small animals? Can J Physiol Pharmacol 76:945-961

12. Onodera M, Kuwaki T, Kumada M, Masuda Y 1997 Determination of ventilatory volume in mice by whole-body plethysmography. Jpn J Physiol 47:317-326

13. Dauger S, Renolleau S, Vardon G, Népote V, Mas C, Simonneau M, Gaultier C, Gallego J 1999 Ventilatory responses to hypercapnia and hypoxia in Mash-1 heterozygous newborn and adult mice. Pediatr Res 46:535-542

14. Dauger S, Nsegbe E, Vardon G, Gaultier C, Gallego J 1998 The effects of restraint on ventilatory responses to hypercapnia and hypoxia in mice. Respir Physiol 112:215 225

15. Tankersley CG, Fitzgerald RS, Mitzner WA, Kleeberger SR 1993 Hypercapnic ventilatory responses in mice differentially susceptible to acute ozone exposure. J Appl Physiol 75:2613-2619

16. Tankersley CG, Fitzgerald RS, Levitt RC, Mitzner WA, Ewart SL, Kleeberger SR 1997 Genetic control of differential baseline breathing pattern. J Appl Physiol $82: 874-881$

17. Tankersley CG, Fitzgerald RS, Kleeberger SR 1994 Differential control of ventilation among inbred strains of mice. Am J Physiol 267:R1371-R1377

18. Tankersley CG, Kleeberger SR, Russ B, Schwartz A, Smith P 1996 Modified control of breathing in genetically obese (ob/ob) mice. J Appl Physiol 81:716-723

19. Pepelko WE, Dixon GA 1975 Arterial blood gas in conscious rats exposed to hypoxia, hypercapnia, or both. J Appl Physiol 38:581-587

20. Powell FL, Wilson WK, Mitchell GS 1998 Time domains of the hypoxic ventilatory response. Respir Physiol 112:123-134

21. Bach KB, Mitchell GS 1998 Hypercapnia-induced long-term depression of respiratory activity requires $\alpha 2$-adrenergic receptors. J Appl Physiol 84:2099-2105

22. Bissonnette JM 2000 Mechanisms regulating hypoxic respiratory depression during fetal postnatal life. Am J Physiol 278:R1391-R1400

23. Eden GJ, Hanson MA 1987 Maturation of the respiratory response to acute hypoxia in the newborn rat. J Physiol 392:1-9
24. Brophy S, Ford TW, Carey M, Jones JFX 1999 Activity of aortic chemoreceptors in the anaesthetized rat. J Physiol 514:821-828

25. Howe A, Pack RJ, Wise JCM 1981 Arterial chemoreceptor-like activity in the abdominal vagus of the rat. J Physiol 320:309-318

26. Horn EM, Waldrop TG 1999 Oxygen-sensing neurons in the caudal hypothalamus: their role in cardiorespiratory control. Respir Physiol 110:219-228

27. Hilton SM 1982 The defence-arousal system its relevance for circulatory respiratory control. J Exp Biol 100:159-174

28. Gulati A, Rebello S, Chari G, Bhat R 1992 Ontogeny of endothelin and its receptors in rat brain. Life Sci 51:1715-1724

29. Iyer RS, Singh G, Rebello S, Roy S, Bhat R, Vidyasagar D, Gulati A 1995 Changes in the concentration of endothelin-1 during development of hypertensive rats. Pharmacology 51:96-104

30. Burton MD, Kawashima A, Brayer JA, Kazemi H, Shannon DC, Schuchardt A, Costantini F, Pachnis V, Kinane TB 1997 RET proto-oncogene is important for the development of respiratory $\mathrm{CO}_{2}$ sensitivity. J Auton Nerv Syst 63:137-143

31. Clouthier DE, Hosoda K, Richardson JA, Williams SC, Yanagisawa H, Kuwaki T, Kumada M, Hammer RE, Yanagisawa M 1998 Cranial and cardiac neural crest defects in endothelin-A receptor-deficient mice. Development 125:813-824

32. Erickson JT, Conover JC, Borday V, Champagnat J, Barbacid M, Yancopoulos G, Katz DM 1996 Mice lacking brain-derived neurotrophic factor exhibit visceral sensory neuron losses distinct from mice lacking NT4 and display a severe developmental deficit in control of breathing. J Neurosci 16:5361-5371

33. Gozal D, Simakajornboon N, Czapla MA, Xue YD, Gozal E, Vlasic V, Lasky JA, Liu JY 2000 Brainstem activation of platelet-derived growth factor-receptor modulates the late phase of the hypoxic ventilatory response. J Neurochem 74:310-319

34. Grasemann H, Lu B, Jiao A, Boudreau J, Gerard NP, De Sanctis GT 1999 Targeted deletion of the neutral endopeptidase gene alters ventilatory responses to acute hypoxia in mice. J Appl Physiol 87:1266-1271

35. Kline DD, Yang T, Huang PL, Prabhakar NR 1998 Altered respiratory responses to hypoxia in mutant mice deficient in neuronal nitric oxide synthase. J Physio 511:273-287

36. Gozal D 1998 Congenital central hypoventilation syndrome: an update. Pediatr Pulmonol 26:273-282

37. Hofstra RM, Valdenaire O, Arch E, Osinga J, Kroes H, Loffler BM, Hamosh A, Meijers C, Buys CH 1999 A loss-of-function mutation in the endothelin-converting enzyme (ECE-1) associated with Hirschsprung disease, cardiac defects, autonomic dysfunction. Am J Hum Genet 64:304-308

38. Amiel J, Salomon R, Attié T, Pelet A, Trang H, Mokhtari M, Gaultier C, Munnich A, Lyonnet S 1998 Mutations of the RET-GDNF signaling pathway in Ondine's curse. Am J Hum Genet 62:715-717

39. Sakai T, Wakizaka A, Matsuda H, Nirasawa Y, Itoh Y 1998 Point mutation in exon 12 of the receptor tyrosine kinase proto-oncogene RET in Ondine-Hirschsprung syndrome. Pediatrics 101:924-925

40. Bolk S, Angrist M, Xie J, Yanagisawa M, Silvestri JM, Weese-Mayer DE, Chakravarti A 1996 Endothelin-3 frameshift mutation in congenital central hypoventilation syndrome. Nat Genet 13:395-396

41. Valdenaire O, Rohrbacher E, Mattei MG 1995 Organization of the gene encoding the human-endothelin-converting enzyme (ECE-1). J Biol Chem 270:29794-29798 